\title{
The Design and Application of System Software about Test Evaluation and Training of the Athlete's Movement Function
}

\author{
Guo Sheng \\ P. E. College, Beihua University \\ Jilin City, Jilin Province, China
}

Keywords: Balance function, Weight training, Interesting training, Gravity track, Visual feedback.

\begin{abstract}
In order to improve the tester's balance control, The balance testing and training system software which can adjust the weight reduction is developed. It can automatically adjust the angle of the training bed and realize the test evaluation of the athlete's balance through the weight reduction experiments on different levels to detect what stress on the feet by using presume sensor combination the data acquisition card. This experiment combined with the virtual technology makes balance training in playing-game way. Weight reduction software can precisely control the balance training data, the results of which are objective and fast and the interface of which is vivid. The evaluation index and every training result can be detected quickly through the athlete's focus track function changed real-time. Assessment software can evaluate the tester's balance control ability fast and accurately, while the virtual game training can stimulate the tester's training enthusiasm.
\end{abstract}

\section{Introduction}

Balance ability is not only an important physiological function, but also on important safeguard for athletes to complete standing, walking and crouching etc. It has been many years since foreign athlete's balance ability test evaluation and training system developed, the advanced products of which are ULtracare and Biodex and so on ,but they are complex to operate and expensive. However, domestic digital equipment development of balance evaluation and training relatively lagged behind, which was limited to simple exercise equipment and basic evaluation. According to the survey. the weight reduction training can greatly increase the training effect for the testers who lack the balance of independent standing, so it is particularly important in the rehabilitation process to develop the system software which will be adjustable and quantitative and objectively reflect the tester's balance capacity. This study based on the computer's rapid data acquisition and real-time processing ability, not only combined with object-oriented programming technology, to get athlete's mechanical parameters of lower limbs under variety of positions, but also combined with graphic and digital real-time display technology, can realize interest training and athlete's balance ability test evaluation operated in virtual environment and weight loss state. The features of this system are as follows: Compared with the embedded balance test system ,the system adopts an updown place machine structure, easier to control; The system uses database to manage tester's information, it is powerful, flexible and convenient, and can be connected with other systems, such as HIS or LTS; The system can control the level of weight loss, it's trajectory smooth; The virtual interesting training of the system has many functions, including game pictures, background music, voice tips and audio feedback etc.

\section{The whole design of the software system}

Based on $\mathrm{VC}++6.0$ development tools, the balance test evaluation and training system software of the athletes in the weight reduction state is written. According to the tester's training and 
requirements for convenient operation, the system is divided into four modules, automatic weight loss control, test evaluation, interesting management. Each module independent seal, Automatic weight loss control module is used to automatically control the tester's weight loss degree, making motor realize the bed-body tilt at an angle. The test evaluation module is used in real-time preservation of the data collected, stress condition of lower limbs, the athlete's gravity track and in outputting evaluation index. Interesting game training module is used in balance training function of lower limbs. This module integrates game animation, background music and voice tip, and testers take pant in game training through force of lower limbs. The tester's information management module manages operating systems, such as adding, correcting and inquiring the tester's basic information. It also includes managing systems, such as assessment data tested many times, evaluation index, training data and training results.

\section{Designing the son module of software system}

Balance ability test evaluation module. This module includes a few son modules, such as signal acquisition, data processing and results showing.

Acquisition of forced signals. Signal acquisition uses PCI-1710 modulus conversion card made in Yanhua company, to collect the simulative signal voltage of foot pressure sensor. The sampling signal mode of PCI-1710 is designed as single end mode which can handle simulative signals from four-way foot pressure sensors. The sample acquisition mode is designed as software timing, that is, it takes 50 milliseconds to collect a group of sensor data. Combined with the secondary setups provided by Yanhua company a special Cpci1710 card is written in order to realize a parade of operational functions, such as the opening and shutting of acquisition card, collecting sample pattern, design of its accuracy and its data storage. First set sampling period of 50 milliseconds for data sampling and record the staring time with an accurate unit(milliseconds). According to management level form a file name including the tester's data of full path. Following that calculate sample total data and apply for its access buffer region. Next open the data a acquisition card to collect data. Set a sampling timing clock to response data sampling, and collect a group of pressure data, converted into voltage value, to show real-time condition of lower limb force. And then, determine the current time, with the unit( milliseconds) .Finally, calculate collecting time (carrying out collecting within the time allowed) and write the data of the buffer region into the file. The data of this system come from foot pressure sensor, and we set them as FA, FB, FC and FD respectively standing for four numeral values of pressure sensors. When testing the static balance ability, we use a calculation formula of athletes' gravity projection position, that is, $(10,11): \mathrm{x}=(\mathrm{FA}+\mathrm{FB}-\mathrm{FC}-\mathrm{FD}), \mathrm{L} / \mathrm{GY}=(\mathrm{FB}+\mathrm{FD}-\mathrm{FA}-\mathrm{FC})$. In M/G formula, L stands a distance between the testing platform center fixed in two pedals and the $\mathrm{x}$ axis of the sensor; $\mathrm{M}$ stands for another distance between the testing platform center fixed in two pedals and the $Y$ axis of the sensor; $G$ is the athlete's weight after weight loss. Foot pressure sensor position distribution can de shown in figure 1.

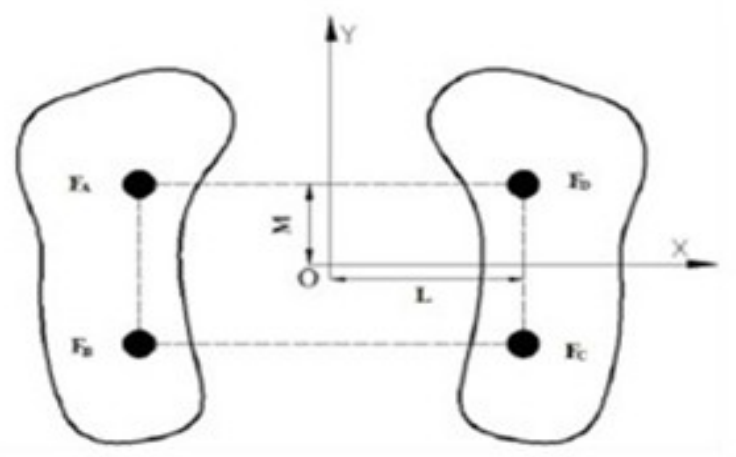

Figure 1. Foot pressure sensor locations 


\section{The calculation method of gravity envelope area in evaluation parameters}

Gravity envelope area is an important index evaluating athletes' balance capacity and the parameters can be got not in weight loss test evaluation process. The calculating process is as follows: First scan all gravity data to determine the minimum rectangle intervals included in gravity distribution diagrams. Following that, draw small rectangular intervals along the $\mathrm{X}$ axis of CG points, put each point into its two interval. The third step is to search two points standing for the maximum $\mathrm{Y}$ and another two points standing for the minimum $\mathrm{Y}$, forming a trapezoid through connecting the four points. Finally, calculate each envelope area in the little interval, and all the trapezoidal area in order to get a total area of gravity moving track.

Data processing. Data structure set in this system can store gravity information, Including gravity coordinate values, interval Numbers index and data chain points' intervals again, we should build up corresponding data link list of each interval, containing relatively less points. The speed of searching four extreme value points of $\mathrm{Y}$ becomes faster, so the complexity of calculating envelope area is reduced. Compare that with the calculating envelope area in convex method, the speed of the former is increased significantly.

Displaying data results. The result calculating module and storage module can make the right and left foot pressure sensor data calculated, and the right and left bar charts real-time displayed on the screen. Because of all data handled in memory, calculating speed is very fast. After the acquisition, according to the tester's name. test type and sampling time, we set up different contents respectively and input the data of memory buffer region into the tester's data files in order to preserve for a long time. The final processing result is out put in WORD document format, which includes the tester's basic information, specific index reflecting the balance capacity and gravity distribution pattern. To conveniently add new functions and upgrade future versions, the data processing operation system is all formed in an AnDatahandle. Dll moving connecting bank. Each evaluation index of each balance is connected to a set of functions, which is convenient and easy to operate. Its implementation process is show in figure 2:

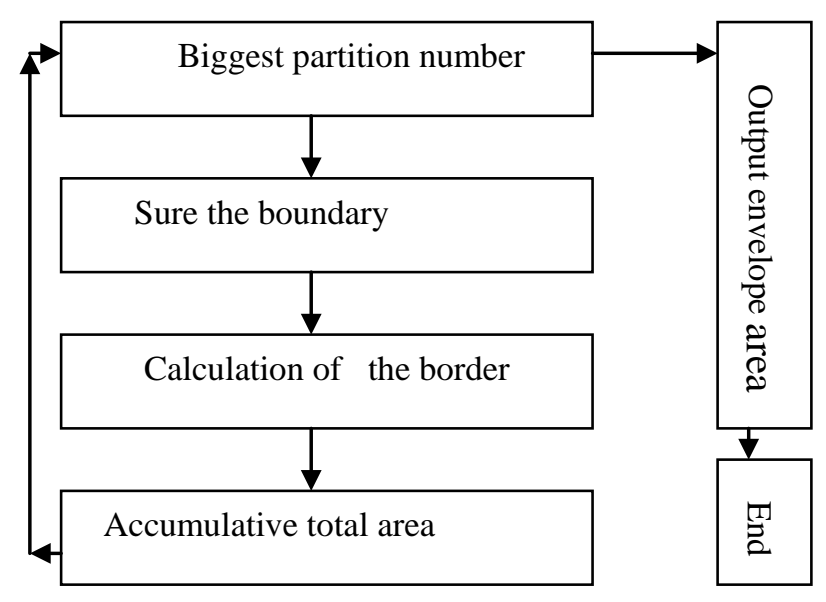

Figure 2. Envelope area computation flow chart

Interesting training module. A game training module of catching fruits is designed in the software, which is combined with a balance rehabilitation training and interesting games to form an interactive training platform including games, visual and auditory feedback. In the balance rehabilitation training, testers make body coordination movement and then their training effects are greatly improved.

Realizing interest training through interest training module. First, open the data acquisition card to collect pressure data. Second, create and initialize game objects. Third, set a clock of fruit creating. If setting renewing fruit position, we should judge whether to create fruit events. If so, it is necessary to create new fruits. We should also judge whether to renew game screen events. If so, we 
calculate new positions of all the fruits and renew game screen. Finally, mobile events are judged. If so, we should observe foot pressure sensor data and judge feet pressure difference in order to decide whether to change car direction.

Operation signal acquisition card. Interesting training state management system, setting system of game difficulty level, background music broadcast system and statistics system of game achievements, all of them are contained in the operation signal acquisition card. Tester's information management system saves all information in data base and data file way. The database is chosen from AACESS database of Microsoft, saving the tester's basic information through tester's basic information table. This system has many functions, such as opening the table, shutting the table, recording, adding, correcting and inquiring etc. When used, it should be connected to database interface. There is no special requirements for database when we install software.

\section{Conclusion}

This system realizes test evaluation of athlete's balance capacity and balance training of weight loss by using motion control, data acquisition, pressure sensor and computer technology, and it accurately controls weight loss degree. There is feedback of voice and vision, lively and interesting interface in balance training process. This system can fast collect and handle data from foot pressure sensors and realize the real-time display of athlete's gravity trajectory and parameter test. It conveniently and accurately tests athlete's balance ability, and improves the accuracy of selecting athletes.

\section{References}

[1]Li Yang "the sport balance function evaluation research progress". Shenyang Sorts institude jurnal.

[2]Li Wenbin, Wang Deming. “The sport balance function test system research progress”. The human Ergonomic.

[3]Zhang Xin "The sport balance function evaluation research progress "Phisical medical learn to learn and rehabilitation.

[4]Wang Jinhe "Athletes focus moved track envelope in the area of the improved algorithm.”Suzhou branch technology university journal.

[5]Li Dan, “Athletes balance ability test algorithm of research”. Tangshan university journal.

[6]Zhang Liyu “A kind of lower limb rehabilitation robots foot force test party method”.Journal of harbin engineering university. 